Cahiers « Mondes anciens »

ANCIENS

Histoire et anthropologie des mondes anciens

3 | 2012

Femmes de paroles

\title{
Voix de femmes songhay-zarma du Niger
}

Entre normes et transgressions

\section{Sandra Bornand}

\section{(2) OpenEdition}

Journals

Édition électronique

URL : http://journals.openedition.org/mondesanciens/675

DOI : $10.4000 /$ mondesanciens. 675

ISSN : 2107-0199

Éditeur

UMR 8210 Anthropologie et Histoire des Mondes Antiques

Référence électronique

Sandra Bornand, «Voix de femmes songhay-zarma du Niger », Cahiers "Mondes anciens » [En ligne], 3 | 2012, mis en ligne le 23 mai 2012, consulté le 19 avril 2019. URL : http://journals.openedition.org/ mondesanciens/675; DOI : 10.4000/mondesanciens.675

Ce document a été généré automatiquement le 19 avril 2019

\section{cc) (†) $\ominus$}

Les Cahiers «Mondes Anciens » sont mis à disposition selon les termes de la licence Creative Commons Attribution - Pas d'Utilisation Commerciale - Pas de Modification 4.0 International. 


\title{
Voix de femmes songhay-zarma du Niger
}

\author{
Entre normes et transgressions
}

Sandra Bornand

1 Chez les Songhay-Zarma du Niger ${ }^{1}$, la polygamie est une pratique courante. Au moment du remariage d'un homme, on observe un rituel spécifique aux mariages polygames - le marcanda - que l'époux offre à celle de ses femmes qui perd son rang de dernière épousée.

2 Je me suis beaucoup intéressée à cette cérémonie (BORNAND 2005, 2010) qui est pour les femmes un moyen socialement toléré de canaliser les conflits que suscite la polygamie. Les femmes mariées s'y retrouvent après la bénédiction du mariage, pour s'affronter de manière essentiellement ludique - bien qu'il puisse y avoir des tensions - lors d'une joute verbale où les premières épousées (les "grandes épouses ») et les suivantes (les « petites épouses ") échangent des insultes ritualisées, dansent et chantent; c'est pour elles un moyen d'exprimer leurs émotions face à une situation qui leur est imposée et sur laquelle elles n'ont pas de prise. Mais ces chants ne possèdent pas qu'une fonction cathartique ; ils expriment parfois - comme nous le verrons - une forme de contestation des normes sociales en vigueur.

3 L'assistance féminine du marcanda permet alors aux femmes mariées d'abandonner la retenue habituelle qu'elles ont en présence des hommes. Il arrive parfois que soient invitées des chanteuses d'origine $"$ captive $»^{2}$. De par leur statut social, leur répertoire est beaucoup plus libéré que celui des chanteuses d'origine libre et l'on peut alors assister à un genre de chants qui évoque, de manière souvent grivoise, provocatrice et jubilatoire, ce dont on ne parle pas au quotidien : la sexualité.

4 Pour comprendre ce genre de performance, il peut être intéressant de le situer dans un ensemble de pratiques mobilisant l'insulte et l'obscénité. C'est le cas, généralement, des relations de "parenté à plaisanterie", étudiées par de nombreux africanistes (cf. notamment CAMARA 1992 ; CANUT et SMITH 2006 ; DIAWARA 1990 et 2003 ; ou encore EVANSPRITCHARD 1929 et RADCLIFFE-BROWN 1940 et 1949). Ainsi, chez les Songhay-Zarma, le cousinage à plaisanterie (baasetaray, litt. : « état de cousins croisés ») peut, comme dans 
d'autres sociétés, dépasser les relations de cousinage au sens strict et "relier deux groupes se rattachant à des ancêtres communs (le frère et la sœur, historiques ou mythiques), [ou] que des péripéties ont rapprochés dans le passé et qui en sont devenus “cousins" au sens figuré » (OLIVIER DE SARDAN 1982, p. 43). D'autres types de relations qui « combinent [...] la familiarité (le jeu) et l'inégalité » (Ibid., p. 48) peuvent être associés au cousinage à plaisanterie; celles, par exemple, liant les grands-parents à leurs petitsenfants ou un " maître » et son captif de case (horso).

Chez les Songhay-Zarma, toutefois, ces relations ne jouent pas, à ma connaissance, sur le registre obscène. La pratique verbale et gestuelle de l'obscénité n'a aujourd'hui plus lieu que dans les marcanda (et encore). Autrefois, elle était aussi le fait de descendants de captifs, et tout particulièrement de ceux que l'on nomme sasaale, des "griotsquémandeurs » dont la fonction principale consistait à insulter et à humilier ceux qui parmi les hommes ou femmes libres se rendaient coupables d'adultère ou d'inceste par exemple ${ }^{3}$.

6 Je montrerai dans cet article, sur la base d'une approche énonciative et pragmatique, comment ces chants de captives obéissent à des normes, bien qu'ils s'inscrivent dans la transgression, et que cet espace transgressif, s'il est délimité, est sans cesse renégocié. C'est pourquoi on peut véritablement parler d'agency ${ }^{4}$ des chanteuses. C'est ce que j'ai pu constater lors d'une séance d'enregistrement en situation "artificielle »: ponctuée de rires et d'anecdotes scabreuses, elle fut brutalement interrompue par la plus âgée des chanteuses, garante, en quelque sorte, de la morale, ce qui déboucha sur une altercation avec l'une de ses co-épouses. Le dernier chant est particulièrement intéressant, car il est celui de la rupture et il permet de comprendre ce qui se joue à ce moment précis.

\section{Un enregistrement particulier}

7 Celui-ci s'insère dans un ensemble de trente-deux chants qui a été enregistré en situation de communication « artificielle» le 3 décembre $2003^{5}$. Leur production étant déjà rare à cette époque, ils sont «venus à moi » par un heureux hasard. Alors que, revenant d'un séjour au village, je rendais visite à une famille que je connaissais, les femmes m'interpellèrent, me demandant ce que j'y avais fait. Je leur racontai les enregistrements de chants de femmes et leur évoquai un marcanda auquel j'avais assisté. L'une d'entre elles me dit alors : «Si tu veux entendre une chose que tu n'as jamais entendue, trouvenous un endroit retiré, loin des hommes ». Intriguée, je dénichai une voiture et obtins le droit d'aller dans un lieu retiré aux limites de Niamey, la capitale du Niger. Deux jours plus tard, quelle ne fut pas ma surprise! Ce que j'enregistrai alors, je ne l'avais effectivement jamais entendu et ne l'ai plus entendu depuis, car cette parole est aujourd'hui interdite par les maris et condamnée par la religion musulmane. Et, chaque fois que les chanteuses pensaient pouvoir m'amener à un marcanda auquel elles étaient conviées, les maris interdisaient au dernier moment leur participation. Depuis, elles ne chantent plus cette version des chants et j'ai probablement assisté à leur dernière prestation.

8 Ont participé à cet enregistrement quatre $\operatorname{coépouses}^{6}(\mathrm{G} 3, \mathrm{G} 4, \mathrm{P} 1, \mathrm{P} 2)$, la nièce de l'une d'entre elles et deux de leurs amies (G5 et G6). Toutes sont d'origine garaasa (groupe des artisans du cuir) ${ }^{7}$ à l'exception de l'une d'elles, issue d'une famille «libre», mais rétrogradée dans la structure sociale songhay-zarma du fait de son mariage. Trois d'entre elles sont originaires de cantons zarma, quatre viennent d'un canton songhay ${ }^{8}$. 
9 La disposition de la performance est la suivante : les deux coépouses les plus âgées sont assises sur une natte. Elles ont installé une bassine d'eau et y ont déposé une calebasse renversée, dont elles se serviront pour rythmer les chants en la frappant d'un bâton. Les chanteuses en revanche se tiennent debout et forment un demi-cercle, placé face à Fati Djida $^{9}$ et moi-même. Celle qui chante s'avance au centre en dansant. Les autres l'accompagnent de battements des mains et ponctuent verbalement ses propos.

10 Si elles agissent de la même manière que lors de performances en situation de communication «habituelle» et si tout montre qu'elles semblent se prendre au jeu et oublier l'artificialité de la situation de communication, la spécificité de cette situation de communication amène toutefois à enfreindre certaines normes du genre discursif. Ainsi, la performance a-t-elle lieu en dehors de toute cérémonie, de jour et en plein air, alors que ces chants sont habituellement chantés dans la maison et de nuit lors du remariage d'un homme. C'est le cadre de ce dispositif particulier qui a permis la présence de femmes célibataires lors de cette performance. En effet, une des chanteuses, Fati Djida, mon assistante et moi-même n'étions pas mariées à l'époque. En contexte "cérémoniel ", aucune d'entre nous n'aurait été autorisée à assister à ces chants.

11 L'enregistrement comprend trente-deux chants. Dès le début, une petite épouse donne le ton avec un chant qui évoque le plaisir féminin lors de l'acte sexuel, mais aussi le désarroi des grandes épouses, qui ne jugent même plus utile de se laver le sexe, tant elles sont délaissées par leur mari. Alors qu'au quotidien on évite de parler des organes génitaux, (à la rare exception de jurons tels que nya dafe, "con de la mère ", baaba bande "bite du père $»)^{10}$, les chanteuses ici se délectent à faire étalage de leurs connaissances lexicales en la matière, passant du familier au vulgaire. Ainsi trouve-t-on les termes suivants : basventre (konye) $)^{11}$, con (dafe), chatte (koosi), foufounette (nyatte), moule (kuna), berlingot (dafayize, kunayize) pour le sexe féminin ; bite (bande, haffu), queue (hanji) ou couilles (fooru, fooriize) pour le sexe masculin. Mais la métaphore est aussi largement usitée. Ainsi, ce dernier est-il canif (santeela), pilon (hinji), poutre (warra) ou encore fourche (ganji) par exemple, tandis que le sexe féminin est petite boîte en plastique (koppiize) ou, quand il a été défloré, passoire (gurbo), trou sans fond (gatamba). Le sperme, quant à lui, n'apparaît que métaphoriquement sous la forme du ragoût d'œufs de gecko (cita gunguri sakku) ${ }^{12}$.

Comme l'écrit CAmara (1992, p. 256) pour les Malinké, mais c'est aussi vrai pour les Songhay-Zarma, nommer les organes génitaux, c'est «exhiber par la parole [son] intimité » et cette publication de l'intimité masculine est un des moyens par lequel les chanteuses vengent la femme dont l'époux se remarie. Plus encore, dans de nombreux chants, elles se moquent de la gent masculine, de ses faiblesses (l'impuissance), de ses défauts physiques (l'homme à un seul testicule), de son manque de retenue et de pudeur (le marabout convoitant la femme d'autrui, tellement obsédé qu'il en renvoie ses élèves ; le beau-père couchant avec sa belle-fille, alors que le fils est parti en exode pour les faire vivre).

13 Ces chants, chantés par d'autres avant elles, mais aussi lieux d'improvisation, leur permettent en outre d'affirmer un désir sexuel et d'évoquer le plaisir qu'elles peuvent éprouver quand leur époux ou - le plus souvent - leur amant est à la hauteur de leurs attentes ${ }^{13}$. Précisons par ailleurs que, dans la réalité, il est recommandé à la femme de ne pas montrer son plaisir. Elles cherchent également par ces chants à montrer qu'elles disposent d'un certain pouvoir sur les hommes. Si de tels chants suscitent les rires parmi les chanteuses, ceux-ci augmentent au fur et à mesure de la performance au point de créer une certaine anarchie. L'engagement des chanteuses dans la performance collective 
va crescendo et atteint son apogée dans le dernier chant, qui se termine par une altercation. Pour calmer le jeu, j'ai alors décidé d'interrompre la séance d'enregistrement.

\section{Un chant qui « dérape »?}

14 Pour comprendre cette altercation, il faut resituer ce dernier chant dans le cadre du marcanda. On a ici affaire à une joute verbale d'insultes sous forme chantée que certaines normes, signalées par les chanteuses elles-mêmes et plus ou moins respectées en pratique, sont censées encadrer : 1 ) une alternance des tours de parole, afin de permettre à chaque groupe de s'exprimer "équitablement»;2) la construction d'une «distance symbolique » qui évite l'adresse directe, notamment par l'interdiction de nommer (seul le groupe est désigné par l'évocation du statut des énonciataires dans leur ménage) ; 3) l'aspect poétique des insultes, que révèlent les figures stylistiques ${ }^{14}$ et la présence de règles linguistiques, participe également de cette distanciation et évite que ces paroles ne mettent en danger l'harmonie sociale.

15 Mais les chanteuses disposent d'une certaine latitude dans leur interprétation de ces règles inhérentes au genre, qui sont donc implicitement renégociées tout au long de la performance ${ }^{15}$. Mon hypothèse est que l'altercation marque une différence d'interprétation, la doyenne des chanteuses reprochant aux autres de faire n'importe quoi (furkutaray), ce à quoi répond sur un ton ferme - marque importante d'irrespect une de ses co-épouses plus jeune.

\section{De la joute « classique » à la joute grivoise}

Ce chant se caractérise par des glissements successifs : un échange d'insultes typiques du marcanda «classique» (que font aussi les femmes d'origine libre) devient chant grivois, une anecdote rompt les règles du genre et enfin une altercation éclate entre deux chanteuses.

Le chant débute par la louange qu'une représentante des petites épouses adresse à son groupe :

(P1) Wande kayney kaayanta

L'arrivée des petites épouses

(P2) Salkatak feeta-feeta

Droites et élancées

(P1) Wande kayney kaayanta salkatak molla-molla danga jeeri ga dira subu ra

L'arrivée des petites épouses droites et élancées à la démarche élégante comme des gazelles marchant dans l'herbe

(P2) Iri wande kayney ma kaani iri wande kayney di yu do

Nous les petites épouses sommes heureuses nous les petites épouses avons trouvé

l'endroit du miel (v. 1-4) ${ }^{16}$

En creux, cet éloge est une critique des grandes épouses, car il sous-entend que celles-ci n'ont pas ces qualités. Cela devient d'ailleurs très vite explicite :

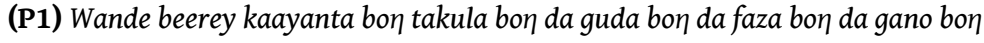

À l'arrivée des grandes femmes la tête pleine de bosses la tête pleine de grumeaux

la tête pleine de poison ${ }^{17}$ la tête pleine de poux [...] (v. 5)

On retrouve un point d'attaque propre aux joutes verbales d'insultes de marcanda, la saleté des grandes épouses ${ }^{18}$, mais un glissement progressif s'opère ici, de la saleté de la tête des grandes femmes à celle du sexe masculin. Le terme bon, qui désigne la tête et plus 
généralement l'extrémité, joue sur l'ambiguïté. Ainsi passe-t-on de la tête des femmes à celle de l'homme, sans que l'on puisse savoir de quelle extrémité on parle :

(G5) Wor ga cim deeda boori zama wor na a gar iri na a hanse cacarma buuna fun bon Vous dites vrai votre récit est bon parce que quand vous l'avez trouvée nous l'avions déjà bien arrangée en enlevant toutes les crasses de la tête (v. 6)

P1 réplique sur le registre sexuel, mais garde encore une certaine ambiguïté :

(P1) Kan wanzamo ${ }^{19}$ na cacarma din ka manti wande beerey ma ga

Quand le wanzam a enlevé toutes ces crasses-là ce n'était pas au nom des grandes épouses

Wande kayney kulu ma ga no i na wanzamo din daך bangu

C'était au nom de toutes les petites épouses qu'on a circoncis le wanzam (v. 9-10)

21 P1 reprend le terme cacarma "crasse » employé par G5 et l'attribue explicitement au wanzam. Par la mention de la "circoncision", l'allusion au sexe masculin se fait plus précise. Mais ici P1 déjoue nos attentes en faisant du « circonciseur » la personne que l'on circoncit; ce qui n'est évidemment pas possible dans les faits, puisqu'il l'aura lui-même été avant son entrée dans le monde adulte. Par cette inversion, elle force à réinterpréter son vers, et la circoncision ne peut être que métaphorique: elle signifie que l'épouse, vieille et laide, n'étant plus désirable, la verge, inutile, était négligée. Le nettoyage est donc un prélude à l'acte sexuel qui redevient envisageable grâce à l'arrivée d'une petite épouse, jeune et désirable. Toutefois, il n'y a dans ce vers aucune prise en charge de l'énonciation et l'acte de circoncision est attribué à un pronom de la $3^{\text {ème }}$ personne indéterminé ( ils, elles, on »).

G5, en reprenant les termes de cacarma et de wanzam, renverse encore l'argument de sa rivale, et rappelle que ce sont les grandes épouses qui initient les hommes à la sexualité, ce dont vont pouvoir bénéficier les petites épouses :

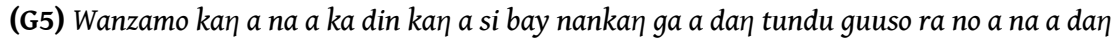
iri no na a dondonandi ga a waan'a waran ma a ka sambu ga a nuusu

Le wanzam quand il l'a sortie comme il ne savait pas où il fallait la mettre il l'a mise dans le trou du cul c'est nous qui lui avons appris jusqu'à ce qu'il maîtrise et vous vous venez vous la prenez et la forcez à entrer (v. 11)

Si le sexe de l'homme reste toujours implicite (emploi du pronom 3S, a), G5 devient plus précise: elle évoque le «trou du cul» (tundu guuso), terme considéré comme particulièrement vulgaire, et assume ses propos (cf. la $1^{\text {ère }}$ personne du pluriel). Tout peut

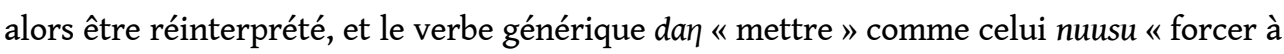
entrer ", désignent clairement l'acte de pénétration. Avec ce dernier, G5 critique au passage les petites épouses qui ne maîtrisent pas les choses du sexe.

\section{De l'évocation du sexe de l'homme à une pratique sexuelle déviante}

Toutefois les chanteuses, prises au jeu, poussent plus loin encore la provocation. Ainsi G5 évoque-t-elle la fellation, pratique sexuelle peu courante et réprouvée, issue, semble-t-il, de la modernité :

(G5) Kwaasay wo wor wanno deede fo bumbo no iri di haafu bon sunsumyan wande kayne jine no

Quant à votre statut de préférées voici une autre histoire nous avons vu que sucer la tête de la bite c'est l'acte des petites épouses (v. 39)

Elle poursuit ses railleries en imaginant les conséquences de cette pratique :

(G5) Haffu bon nano din ra han fo wor ga a dagu 
C'est en suçant cette tête de la bite-là qu'un jour vous allez l'arracher (v. 59) épouse ; un jour, sa grande épouse - jalouse des privilèges de sa rivale - exigea de le faire et en profita pour lui couper les testicules. Cette anecdote a suscité les rires des autres femmes, puis P2 a repris le fil et P1 - représentante des petites épouses - a réintégré, par un procédé de glissements successifs, cette anecdote dans le chant :

(P2) Wande beeri si waan'a no wande beeri si waan'a wo din se a go ga futu

La grande épouse ne sait pas le faire la grande épouse ne sait pas le faire c'est pour cela qu'elle est fâchée

(P1) A si waan'a no a si waan'a no a mana a dondon

Elle ne sait pas le faire elle ne sait pas le faire elle ne l'a pas appris

(P2) Wo din se no a go ga futu

C'est pour cela qu'elle est fâchée

(P1) A si waan'a no a si waan'a no iri yeesey din boro si waan'a fa

Elle ne sait pas le faire elle ne sait pas le faire nos manières-là elle ne sait pas les faire

(P1) A si waan'a no a mana a dondon a si waan'a no a mana a dondon a mana a dondon se no a na zaama dan ga dumbu se no a siino dan ga nyalle

Elle ne sait pas le faire elle ne l'a pas appris elle ne l'a pas appris c'est pour cela qu'elle les a coupées avec un couteau c'est pour cela qu'elle les a déchirées avec un rasoir (v. 69-73)

P1 reprend le vers précédant l'anecdote, répétant à l'envi l'incompétence sexuelle des grandes épouses, puis réintroduit des éléments de l'anecdote dans la joute (cf. v. 73), ce qui lui permet de se moquer de l'époux ainsi émasculé :

Mayjida ye zaara fakayan

Monsieur doit porter un pagne en bandoulière (v. 74)

Cette fois, c'est le chant qui va amener à repréciser l'anecdote. S'appuyant sur les conséquences de l'émasculation (l'époux ne supporte plus le pantalon et doit se contenter d'un pagne en bandoulière, équivalent de notre robe de chambre qu'on ne porte que dans l'intimité), la femme évoque le stationnement prolongé de la voiture devant la maison du malheureux. L'association d'idées entre la présence immobile de ce véhicule, car l'homme ne peut sortir habillé en pagne, et la cause de celle-ci (l'émasculation) provoque les fous rires des chanteuses. Ces interactions - comme d'autres tout au long de ce chant peuvent être perçues comme une forme de contestation dans le cadre des relations de pouvoir, que celles-ci relèvent de la hiérarchie entre hommes et femmes ou de celle entre «petites » et « grandes » épouses.

S'élève alors, en arrière-plan, une voix jusque-là discrète qui dit, d'un ton fâché :

Wa te dooni hanno da furkutaray 
Faites de beaux chants au lieu de ce dévergondage [de cette indécence] comportement contraire à la morale, il exprime un jugement sans appel. Ainsi G3 condamne-t-elle la prestation des chanteuses et notamment d'une de ses rivales au sein du foyer. Cette rivalité est clairement ressentie par P1, qui était alors en train de chanter, et qui réplique vexée :

Furkutaray zaati manti furkutaray no wallayi hay!

Comment ça du dévergondage au nom de Dieu ce n'est pas du dévergondage ben non!

L'échange n'est pas anodin, car il met aux prises les épouses d'un même foyer; la « petite » ne respecte pas comme elle le devrait une femme, plus âgée qu'elle, et, de plus, la première épouse de leur mari. La réplique, ainsi que le ton sur lequel elle est prononcée, marquent une perte de maîtrise de soi, indice que quelque chose d'important est en train de se jouer... Même si l'altercation est brève (elle tient sur deux phrases), elle est d'une importance capitale pour comprendre les enjeux de cette performance particulière, mais plus encore des chants de marcanda en général.

\section{Hypothèses sur l'altercation}

Quatre hypothèses peuvent, à mon avis, expliquer l'intervention de G3 qui, en tant que première femme, est la dépositaire de l'autorité familiale en l'absence de l'époux : 1) une perte de maîtrise dans l'interaction ; 2) une énonciation trop directe ; 3) une rupture de registre dans le marcanda et 4) l'évocation d'une pratique sexuelle déviante.

\section{Une perte de maîtrise dans l'interaction?}

Ce chant se présente sous la forme d'une joute, même s'il n'y a pas alternance systématique des représentantes des petites et grandes épouses. Cinq chanteuses fonctionnent ici comme solistes : deux appartiennent au groupe des petites épouses (P1 \& P2) et quatre aux grandes épouses (G3, G4, G5 \& G6).

Sur les 75 vers, 57 sont chantés par des petites épouses (45 par P1, 12 par P2). Le groupe des grandes épouses est représenté par G5 qui chante 15 des 18 vers. Ainsi ce chant consiste principalement dans l'affrontement de deux chanteuses, P1 et G5 qui ne sont pas co-épouses mais amies dans la vie. Dans ce cadre, la norme des joutes qui veut que deux co-épouses ne s'affrontent pas directement est respectée.

ésitations provoquées par les rires commencent au vers 11. P2, perturbée par les autres qui pouffent, ne peut que répliquer mollement et doit se reprendre à deux fois pour terminer son vers. Dès lors, les rires deviennent plus fréquents, rendent parfois les propos inaudibles et perturbent les solistes qui n'arrivent plus à terminer leur vers ou en perdent le fil.

À mesure que le chant se déroule, les enchaînements des tours de parole deviennent plus anarchiques, notamment par l'empressement de P5 qui est seule à véritablement défendre les grandes épouses. Elle se déchaîne et anticipe ses répliques au point que les autres lui enjoignent de se calmer un peu en lui disant hangan hangan (" écoute, écoute"; cela veut dire aussi « attends, attends »). Mais cela ne dure pas, car elle interrompt encore le chant pour narrer l'anecdote évoquée ci-dessus. Cette anecdote, bien qu'elle s'inscrive parfaitement dans la thématique du chant, marque une rupture formelle. Elle entraîne les 
rires des autres chanteuses. Le chant reprendra, mais des rires et un complément à l'anecdote l'interrompra cinq vers plus tard, au moment où P1 se moque de l'homme :

(P1) Mayjida ye zaara fakayan

Monsieur doit porter un pagne en bandoulière (v. 74)

C'est à ce moment qu'intervient l'altercation entre G3 et sa coépouse. Cette anarchie peut être une des raisons qui pousse à l'interruption, mais la virulence de cette dernière ne peut être expliquée par ce seul facteur. En effet, les performances en situation de communication « cérémonielle » connaissent souvent une telle pagaille.

\section{L'évocation d'une pratique sexuelle déviante?}

Si tous les chants sont grivois, si tous - à l'exception de ceux chantés par la "nièce " d'une des chanteuses - évoquent le sexe, sujet tabou par excellence dans la société songhay-zarma, ils n'évoquent pas des pratiques indicibles. Or, le trente-deuxième chant se caractérise par l'irruption d'une pratique sexuelle moderne, apparemment considérée comme déviante, la fellation (haafu ${ }^{21}$ bon sumsumaya : « sucer la tête de la bite », v. 39 ).

Les quatre termes qui, associés au terme haafu «bite » (v. 39), désignent cette pratique

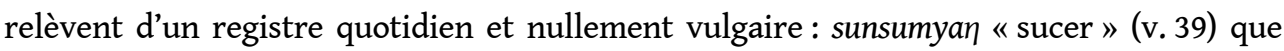
l'on peut tout à fait employer pour un bonbon, naano « tétée » $(v .58,59)$ désigne surtout

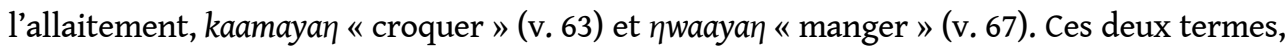
associés à «bite», provoquent l'hilarité des chanteuses. La fellation n'est pas une pratique sexuelle courante et «traditionnelle », mais attribuée à la " civilisation » (v. 45 : cibilizatiyõ). Cette évocation est probablement inspirée des photographies et films pornographiques, rapportés notamment par les hommes qui partaient en exode au Ghana et en Côte d'Ivoire.

Accusées de pratiquer cet acte, les petites épouses répliquent non pas en niant, mais en le revendiquant, le qualifiant de « beau » (v. 52 : hanno), agréable ${ }^{22}$ (v. 66). Elles décrivent en détail leur pratique ${ }^{23}$ (v. 58) :

(P1) [...] yo ziyan ay toola-toola no ga bisa ay mooru-mooru ... haffu bon naano no ga bisa zama ay kwaasay no kand'a ay ma kolli ay ma a ganday ay ma a mooru ay ma a ganday

[...] Ma douceur et mes petites caresses valent mieux que donner des coups de pieds de chameau ... il vaut mieux téter la tête de la bite car je suis la plus aimée je la tire je l'enlace je la caresse je l'enlace 
non seulement elle n'interrompt pas la performance à ce moment, mais - en plus - d'une voix discrète elle chante « ce sont les petites épouses qui le font ».

\section{Une énonciation trop directe?}

Lors d'un marcanda, on observe une oscillation entre une mise à distance et une prise en charge de l'énonciation. Habituellement, la joute tend vers la mise à distance, alors que les chants mettent en scène une plus grande prise en charge par l'emploi des pronoms de $1^{\text {ère }}$ personne (BORNAND 2005, 2010). Si la mise à distance permet d'éviter une confrontation directe, source potentielle de conflit, la prise en charge a pour fonction de créer l'empathie et d'accompagner la femme pour qui est organisé le marcanda dans son deuil de dernière épousée.

Dans le déroulement de la joute, on remarque une oscillation entre énoncés gnomiques et prise en charge. Ainsi, l'absence de traces énonciatives est remarquable dans les trois premiers vers, uniquement constitués de syntagmes nominaux :

(P1) Wande kayney kaayanta

L'arrivée des petites épouses

(P2) Salkatak feeta-feeta

Elancées et élégantes

(P1) Wande kayney kaayanta salkatak molla-molla danga jeeri ga dira subu ra

L'arrivée des petites épouses élancées à la démarche douce comme une gazelle marchant dans l'herbe (v. 1-3)

La subjectivité de l'éloge transparaît uniquement à travers les termes employés, ici une métaphore et une comparaison élogieuse. Ainsi les idéophones feeta-feeta et salkatak caractérisent-ils la beauté de la femme, son physique élancé, une position debout, droite et fière. Quant à l'expression molla-molla, elle procède par métaphore : elle vient, en effet, de mollo qui désigne un lézard de couleur jaune-marron, réputé pour sa finesse et sa beauté. En complétant cette métaphore par la comparaison avec la gazelle, animal à la démarche souple, élégante et légère, la soliste renforce l'éloge.

C'est à partir du quatrième vers qu'apparaissent des pronoms marquant la prise en charge et que se construit une opposition entre " grandes » et « petites » épouses :

(P2) Iri wande kayney ma kaani iri wande kayney di yu do

Nous les petites épouses sommes heureuses nous les petites épouses avons trouvé

l'endroit du miel (v. 4)

(G5) Wor ga cim deeda boori zama wor na a gar iri na a hanse cacarma buuna fun bon ga

Vous dites la vérité le récit est bon parce que vous l'avez trouvée après que nous l' avons bien arrangée en enlevant toutes les crasses de la tête (v. 6)

51 Dans la plupart des joutes entendues, les insultes ne sont pas prises en charge par l'énonciatrice et l'allusion au groupe rival se fait plutôt par sa désignation : « les grandes épouses"vs «les petites épouses ». Les énoncés gnomiques permettent aux solistes d'exprimer un propos subversif sans pour autant avoir à l'assumer, tout en le posant comme une vérité incontestable :

(G3) Wande kayney no ga a te

Ce sont les petites épouses qui le font (v. 40)

Mais ici, les solistes prennent très souvent en charge l'énonciation, d'abord par l'emploi de pronoms de $1^{\text {ère }}$ et $2^{\text {ème }}$ personne du pluriel pour renvoyer à leur groupe d'appartenance, mais - fait plus rare (et implicitement proscrit) - également aux $1^{\text {ère }}$ et 2 ème personnes du singulier. 
$53 \mathrm{Au}$ début, ceux-ci apparaissent dans des discours rapportés, comme cela se fait « classiquement », signalant que la prise en charge est jouée et qu'il y a foisonnement des voix énonciatives :

(P1) [...] a ne ay si gani wi boro kulu se da ay na a wi ay kambey ma goffi

[...] elle dit : «je ne vais pas tuer de poux pour qui que ce soit si j'en tue que mes doigts attrapent la lèpre! » (v. 5)

Mais quand la tension monte, P1, puis P2 s'affranchissent du cadre du discours rapporté pour s'exprimer à la $1^{\text {ère }}$ personne du singulier et opérer de fait, même s'il y a théâtralisation (cf. l'apostrophe « femme »), une prise en charge plus personnelle :

(P1) Ye fu (P1+P2) ga goro (P1) Waybora (P1+P2) hay ye ga ka ga goro / (P1) ye ga ka ye day ga goro ay ya si dira ay si miiri [hésitations] ne [...] tiri-tiri no gokke

Retourne à la maison! Femme! Eh! Retourne et reste-s'y! Retourne et reste-s'y vraiment ! Quant à moi je ne bougerai pas d'ici je m'enfoncerai dans la terre [...] le ventre bombé très tendu (v. 30)

Plus encore, P1 va jusqu'à évoquer la fellation et le plaisir qu'elle y prend à la $1^{\text {ère }}$ personne :

(P1) [...] guna yo ziyan ay toola-toola no ga bisa ay mooru-mooru ... haffu bon naano no ga bisa zama ay kwaasay no kand'a ay ma kolli ay ma a ganday ay ma a mooru ay ma a ganday

[...] Regarde! Ma douceur et mes petites caresses valent mieux que donner des coups de pieds de chameau ... il vaut mieux téter la tête de la bite car je suis la plus aimée je la tire je l'enlace je la caresse je l'enlace (v. 58)

Qui se cache derrière ce « je » ? La soliste elle-même ou la locutrice-petite épouse qu'elle a installée ? Comment penser que P1 puisse assumer de tels propos face à moi, alors que je connais très bien son époux ? Si dire « je » dans un tel cadre crée une ambiguïté, il n'est pas certain que le seul fait de chanter suffise à maintenir l'effet de théâtralisation. J'avais signalé ailleurs (BORNAND 2005) que les énoncés gnomiques ainsi que les nombreuses figures stylistiques permettaient de mettre à distance les propos et éviter les interprétations personnelles. Or, ici, nous nous retrouvons face à des emplois de la $1^{\text {ère }}$ et de la $2^{\text {ème }}$ personne du singulier et à des propos explicites, y compris pour des pratiques sexuelles comme la fellation. Cette prise en charge, toujours plus forte, pourrait être une des causes de l'altercation. Mais comme G3 n'intervient pas immédiatement après, on peut en déduire que la mise en scène de la subjectivité en elle-même ne suffit pas non plus à expliquer, à elle seule, l'altercation.

58 Est-ce alors le terme hausa de mayjida ("Monsieur », litt. " possesseur de la concession »), mentionné une quinzaine de vers plus loin, qui est de trop? Ce terme est utilisé comme marque de respect par les épouses qui n'ont pas le droit de prononcer le nom de leur mari et, plus généralement, pour désigner avec déférence un chef de famille :

(P1) A si waan'a no a mana a dondon'a si waan'a no a mana a dondon a mana dondon se no

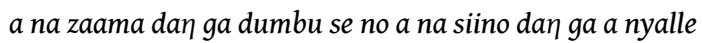

Elle ne le sait pas elle ne l'a pas appris elle ne le sait pas elle ne l'a pas appris elle ne l'a pas appris c'est pour cela qu'elle l'a coupée c'est pour cela qu'elle l'a déchirée avec le rasoir

(P1) Mayjida ye zaara fakayan

Monsieur doit porter un pagne en bandoulière

(P1) Mayjida ye zaara fakaya

Monsieur doit porter un pagne en bandoulière (v. 73-75)

59 Or la confrontation de cette marque de respect avec l'évocation d'une situation humiliante (sexualité déviante, voire d'une émasculation) est perçue comme un affront 
intolérable à l'homme dans sa fonction d'époux et de chef de famille. Pour G3, nous ne sommes probablement plus ici dans le jeu... et c'est peut-être pour éviter tout débordement supplémentaire qu'elle intervient juste après l'évocation du mayjida.

\section{La rupture $d u$ registre $d u$ marcanda?} particulier : l'événement mentionné est situé dans un quartier spécifique de Niamey et l'homme désigné est supposé avoir deux épouses et posséder une voiture, ce qui est suffisamment particulier pour permettre son identification dans l'hypothèse que ce personnage existe vraiment. Ce risque de dévoilement de situations intimes, de tiers que l'assemblée pourrait reconnaître, explique en partie l'interruption de G3.

\section{Conclusion}

62 Cette performance fut surprenante à plus d'un titre. Non seulement les chanteuses me firent découvrir des chants que je n'avais jamais entendus et qu'elles n'ont, depuis, plus eu l'occasion de chanter, mais la performance se termina par une altercation, me donnant ainsi l'occasion d'analyser les règles inhérentes à ces chants pourtant très transgressifs, des règles souvent implicites et sans cesse renégociées au cours de la performance. En

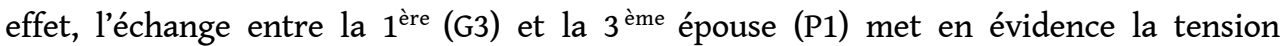
présente à ce moment ; si ce genre de conflit se règle idéalement en privé, sans témoins, il arrive néanmoins qu'ils éclatent au quotidien ou en situation "cérémonielle " de marcanda.

63 Ces chanteuses, d'origine "captive ", ont cette spécificité de pouvoir se montrer très impudiques, alors même que la pudeur est au cœur de la société songhay-zarma. En abordant la sexualité, sans tabou, elles jouent un jeu dangereux, car la performance peut vite être interprétée comme inadéquate, surtout dans le contexte actuel de forte islamisation. C'est d'ailleurs la raison pour laquelle elles sont encore invitées mais souvent interdites de parole. ce qui apparaît tout particulièrement dans le dernier chant énoncé sous forme de joute : les solistes, emportées par leur fougue, vont toujours plus loin dans les provocations, jusqu'à l'intervention de G3, qui qualifie, d'un ton fâché, leur prestation par le terme très fort de furkutaray ( dévergondage »). interpréter comme potentiellement dangereux pour l'harmonie sociale, la cohésion et la réputation familiale :

66 - Le glissement de l'évocation de la sexualité (acceptable pour des femmes d'origine " captive ») à une sexualité déviante, la fellation, évoquée finalement à la $1^{\text {ère }}$ personne du singulier, et, en outre, avec une certaine gourmandise. 
67 - Le glissement d'une qualification «grandes épouses»vs «petites épouses» (norme valorisée) à un « nous » vs « vous » (norme acceptable), puis à un « je » vs « tu » qui tend à effacer la distance symbolique et à prendre le risque d'une confrontation personnelle.

68 - L'anecdote qui interrompt la joute par le changement de registre (du chanté au parlé) confirme la crainte de la personnalisation.

69 - Le glissement d'un terme extrêmement respectueux mayjida (qui sert souvent à désigner son propre époux) vers la situation humiliante dans laquelle l'homme est placé et les rires débordants que provoque cette évocation chez les chanteuses constituent un affront intolérable, même si la situation reste imaginaire. Car le risque est grand que les personnes présentes puissent effectuer un rapprochement entre ces hommes dont on se moque et l'époux commun de quatre de ces femmes.

70 En intervenant sur le registre du parlé et non du chanté, Hajia marque l'interruption de la performance, afin que les chanteuses ne dérapent pas. Son intervention se justifie par son âge, son rang de première épouse et le pèlerinage (haj) qu'elle a effectué à la Mecque. Dépositaire en l'absence du mari de l'autorité et de la réputation familiale, caution morale au niveau religieux, c'était donc à elle de restreindre la fougue des « jeunes ».

71 La réplique cinglante de la petite épouse montre que celle-ci n'est pas sur la même longueur d'ondes et signale une différence d'interprétation à propos de la performance. Bien qu'habituées à vivre et à chanter ensemble, G3 et P1 ne ressentent ni ne comprennent de la même manière ce qui se déroule à ce moment-là de la performance. L'altercation prouve que cette limite entre norme et transgression est floue, que les frontières du genre sont mouvantes et, par conséquent, que la négociation implicite a échoué. Aux voix énonciatives féminines présentes dans le chant se mêlent donc des voix individuelles, celles de femmes bien réelles, et l'altercation montre qu'il est parfois difficile de faire la part des choses entre cette polygamie que l'on chante et celle que l'on vit.

\section{BIBLIOGRAPHIE}

ADAM J.-M. (1999), “ “Ta mère...” : de l'insulte à l'histoire drôle » dans Adam J.-M., Linguistique textuelle. Des genres de discours aux textes, Paris, p. 157-173.

ANSCOMBRE, J.-Cl. (2006), « Stéréotypes, gnomicité et polyphonie : La voix de son maître », dans $\mathrm{P}$ errin L. éd., Le sens et ses voix. Dialogisme et polyphonie en langue et en discours, Recherches linguistiques 28, Metz, p. 349-378.

BERNARD Y. et WHITE-KABA M. (1994), Dictionnaire zarma-français (République du Niger), Niamey-Paris. BISILLIAT J. et LAYA D. (1992), « Le système familial songhay-zarma », Journal des Africanistes 62 (2), p. 161-181.

BORNAND S. (2005), Le Discours du griot généalogiste chez les Zarma du Niger, Paris. 
BORNAND S. (2005), « Insultes rituelles entre coépouses. Étude du marcanda (Zarma, Niger) », ethnographiques.org, [En ligne], 7 | 2005. Consulté le 24.02.2012. URL : http:// www.ethnographiques.org/2005/ Bornand BORNAND S. (2006), Parlons Zarma. Une langue du Niger, Paris.

BORNAND S. (2008), « Le marcanda ou le deuil de la femme préférée », dans Abomo-Maurin M.R. éd., Littérature orale, genres, fonctions et réécriture, Paris, p. 71-82.

BORNAND S. (2010), «Chants de douleur des femmes zarma (Niger) », dans Calame C., Dupont F. et Manca M. éd., La Voix actée, Paris, p. 159-173.

BOUTET J. (2010), Le pouvoir des mots, Paris.

CAMARA S. (1992), Gens de la parole : essai sur la condition et le rôle des griots dans la société malinké,

Paris.

CANUT C. (2002), « Pouvoirs, places et filiation. Les Senankuya en milieu urbain au Mali », Cahiers de Praxématique 38, p. 175-197.

CANUt C. et smith E. (2006), " Pactes, alliances et plaisanteries. Pratiques locales, discours global », Cahiers d'études africaines 184, « Parenté, plaisanteries et politique », p. 687-754.

DERIVE J. (2004), « Le bàra : analyse de la naissance d'un genre chez les Dioula de Kong » dans Dauphin A.M. et Derive J. éd., Oralité africaine et création, actes du colloque international de l'Isola 2002, Paris, p. 849-856.

DERIVE J. (2005), « Jeux gratuits, jeux payants : jeux et enjeux de la parodie dans une société africaine ", Le jeu, Chambéry, p. 23-33.

DERIVE J. et DERIVE M.-J. (2004), « Processus de création et valeur d'emploi des insultes en français populaire de Côte-d'Ivoire ", Langue française vol. 144, p. 13-34.

DIALLO Y. (2006), «Identités et relations de plaisanterie chez les Peuls de l'ouest du Burkina Faso, Cahiers d'Études Africaines 184, « Parenté, plaisanteries et politique », p. 779-794.

DIARRA F. A. (1971), Femmes africaines en devenir, les femmes zarma du Niger, Paris.

DIAWARA M. (1990), La Graine de la Parole, Stuttgart.

DIAWARA M. (2003), L'empire du verbe et l'éloquence du silence, Köln.

DUCROZ J.-M. et CHARLES M.-C. (1982), L'homme songhay tel qu'il se dit chez les Kaado du Niger, Paris.

DUPIRE M. (1979), « Obscénité et société virelangues Serer (Senegal) », Research in African Literatures 10 (1), p. 75-89.

DURANTI A. (2009), « Agency in Language » dans Duranti A. éd., A Companion to Linguistic Anthropology, Oxford, Victoria, p. 105-134.

EVANS-PRITCHARD E.E. (1929), « Some Collective Expressions of Obscenity in Africa », Journal of the Royal Anthropological Institute 49, p. 311-331.

FOUÉRÉ M.-A. (2008), Les relations à plaisanteries en Afrique (Tanzanie). Discours savants et pratiques locales, Paris.

GADO B.A. (1980), Le Zarmatarey. Contribution à l'histoire des populations d'entre Niger et Dallol Mawri, Etudes Nigériennes 45, Niamey.

GOFFMAN E. (1974), Les rites d'interaction, Paris. 
GOFFMAN E. (1987), Façons de parler, Paris.

HALE Th. A (1998), Griots and Griottes, Bloomington.

HAMA B. (1988), L'essence du verbe, Niamey.

HEATH J. (1998), Dictionnaire Songhay-Anglais-Français, vol. 3, Koroboro Senni, Paris.

HEATH J. (1999), A Grammar of Koyraboro (Koroboro) Senni, Köln.

IDRISSA K. (1997), « Ethnicité, politique et démocratie au Niger », Sociétés africaines et diaspora 5, p. 45-72.

IRVINE J. (1994), « Insult and Responsibility : Verbal Abuse in a Wolof Village » dans Hill J. \& Irvine J. éd., Responsibility and Evidence in Oral Discourse, Cambridge, p. 105-134.

ISUFI A.U. (1999), Zarma ciine kaamuusu kayna, Niamey.

LABOV W. (1972), Le Parler ordinaire, Paris.

LABOURET H. (1929), « La parenté à plaisanteries en Afrique Occidentale », Africa 2, p. 244-254.

LALLEMAND S. (1975), “"Têtes en loques” : insulte et pédagogie chez les Mossi », Cahiers d'Etudes africaines 60, vol. XV (4), p. 649-667.

LARGUECHE E. (1997), Injure et sexualité. Le corps du délit, Paris.

LARGUECHE E. (2009), Espèce de... ! Les lois de l'effet injure, Chambéry.

LAUNAY R. (2006), « Practical Joking », Cahiers d'études africaines 184, « Parenté, plaisanteries et politique », p. 795-808.

MAUSS M. (1928), « Parentés à plaisanterie ", Annuaire de l'Ecole pratique des hautes études, Section des sciences religieuses («Les classiques en sciences sociales »), p. 3-21.

MCNAUGHTON P.R. (1988), The Mande Blacksmiths: Knowledge, Power and Art in West Africa, Bloomington.

MOUNKAÏLA F. (2008), Anthologie de la littérature orale songhay-zarma. Tome 1. Les textes circonstanciels (appels et mythes d'origine), Paris.

MOUNKAÏLA F. (2008), Anthologie de la littérature orale songhay-zarma. Tome 2. Chants d'intégration sociale, Paris.

MOUNKAÏLA F. (2008), Anthologie de la littérature orale songhay-zarma. Tome 3. Histoire éthique et idéal. Chroniques, épopées, contes et fables édifiantes, Paris.

MOUNKAÏLA F. (2008), Anthologie de la littérature orale songhay-zarma. Tome 4. Textes récréatifs : chants et histoires d'amour, formes théâtralisées des aires de jeux et danses, critique sociale indirecte, Paris.

OLIVIER DE SARDAN J.-P. (1973), « Personnalité et structure sociale », dans Dieterlen G. éd., La notion de personne en Afrique noire, Paris, p. 421-445.

OLIVIER DE SARDAN J.-P. (1982), Concepts et conceptions songhay-zarma. Histoire culturel société, Paris. OLIVIER DE SARDAN J.-P. (1984), Les sociétés songhay-zarma (Niger-Mali). Chefs, guerriers, esclaves, paysans..., Paris.

OLIVIER DE SARDAN J.-P. (2000), « Unités et diversité de l'ensemble songhay-zarma-dendi » dans Soumouni E., Gado B. \& Olivier de Sardan J.-P. éd., Peuplement et Migrations, Actes du premier colloque international 26-29 septembre 1995, Parakou, Niamey, p. 75-98. 
RADCLIFFE-BROWN A.R. (1940), « On Joking Relationships », Africa 13 (3), p. 195-210.

RADCLIFFE-BROWN A.R. (1949), « A further Note on Joking Relationships », Africa 19, p. 133-140.

RICHARDS A.I. (1937), « Reciprocal Clan Relationships among the Bemba of N.E. Rhodesia », Man 37, p. $188-193$.

RIESMAN P. (1974), Société et liberté chez les Peuls Djelgôbé de Haute-Volta. Essais d'anthropologie introspective, Paris.

RODEGEM F.M. (1976), « Les injures agonistiques des gardiens de bétails au Burundi », Psychopathologie africaine vol. XII (3), p. 413-442.

ROUCH J. (1989), La Religion et la magie songhay, Bruxelles.

SALL I.A. (2004), « Licence verbale et mouvements contestataires chez les Haal pulareebe du Fuuta Tooro : Almudaagal ngay et Cooloyaagal », REMMM 103-104, p. 201-221.

SISSAO A.J. (2002), Alliances et parentés à plaisanterie au Burkina Faso. Mécanismes de fonctionnement et avenir, Ouagadougou.

SMITH E. (2004), « Les cousinages de plaisanterie en Afrique de l'Ouest, entre particularismes et universalismes », Raisons politiques 13, p. 157-169.

Sow I. (1991), « Réflexions sur les injures et paroles obscènes au Sénégal », Bulletin de l'Institut fondamental d'Afrique Noire, série B, 46 (3-4), p. 343-378.

TAMARI T. (1993), «L'alliance à plaisanteries interclanique (senankunya) : un instrument de la diplomatie mandingue traditionnelle ", document présenté à l'International Conference for Mande Studies, 15-19 Mars, Bamako.

TERSIS N. (1981), Economie d'un système. Unités et relations syntaxiques en zarma (Niger), Paris.

VetToRato C. (2008), Un Monde où l'on clashe. La joute verbale d'insultes dans la culture de rue, Lyon.

WENDLING T. (2005), «Perspectives comparatives sur les joutes oratoires », ethnographiques.org, [En ligne], 7 | 2005. Consulté le 24.02.2012. URL : http://www.ethnographiques.org/2005/ wendling

\section{NOTES}

1. La région songhay-zarma se trouve à l'Ouest du Niger. Les Songhay sont aussi présents au Mali, alors que les Zarma habitent uniquement le Niger. Si les membres de leur aristocratie revendiquent un ancêtre différent, la langue et le patrimoine socio-culturel mettent en évidence une certaine unité de l'ensemble songhay-zarma.

2. La société songhay-zarma est traversée, depuis l'époque pré-coloniale, par une dichotomie structurale profonde entre hommes libres (burcin, souvent appelés «nobles» en français nigérien) et captifs. Les captifs (bannya) étaient des prises de guerre de l'époque pré-coloniale qui pouvaient être maltraités et vendus. Au centre de cette dichotomie, qui sous-tend aujourd'hui encore l'ensemble du tissu social et justifie a priori le maintien au pouvoir de quelques familles nobles, se trouve le sentiment de honte (haawi : terme polysémique désignant tant la pudeur que la gêne, la bienséance ou le savoir-vivre) que ne connaîtraient que les hommes libres. Cette dichotomie noble-captif se marque donc unilatéralement par la valorisation des uns et la discrimination des autres; " "captif" connot[ant] le vulgaire, le déraciné, il est associé à la 
faiblesse de caractère ou à l'absence de vergogne, à la grossièreté ou aux manquements au savoirvivre » (OLIVIER DE SARDAN 1984, p. 29).

3. Signalons que dans la société malinké - qui possède de nombreux points communs avec les Songhay-Zarma - les " cousins à plaisanterie » " peuvent échanger publiquement des injures et des grossièretés [notamment liées au sexe] qui, en d'autres circonstances, provoqueraient des querelles graves» (CAMARA 1992, p. 43). L'énonciation de grossièretés, sans rapport avec la question de la parenté à plaisanterie, est également attestée lors du solisi (danses nocturnes précédant la circoncision) où « les jeunes gens sillonnent le village proférant des paroles dont le caractère grivois reste masqué par le bruit des tambours et la discordance des voix » (Ibid.: 193), loin des personnes âgées. On retrouve ici un point commun avec le marcanda étudié dans cet article : le cadre rituel et l'éloignement de ceux qui ne doivent pas entendre.

4. Je reprends à mon compte la définition de travail établie par DURANTI $(2009$, p. 453): «1) Agency is here understood as the property of those entities (i) that have some degree of control over their own behavior, (ii) whose actions in the world affect other entities (and sometimes their own), and (iii) whose actions are the object of evaluation (e.g. in termes of their responsibility for a given outcome) ».

5. En situation de communication "artificielle », l'énonciateur profère un discours en privé. Outre l'absence de l'énonciataire «traditionnel », le temps et le lieu de l'énonciation ne sont donc pas respectés. Cette stratégie, systématiquement mise en place lorsqu'il est impossible de réaliser un enregistrement en situation de communication « habituelle» ou provoquée, est à distinguer de cette dernière où la performance respecte les paramètres spatio-temporels (hors cérémonie), l'énonciateur procédant, sur ma demande, comme à son habitude. Bien qu'en cela plus proche de la situation de communication « habituelle ", elle reste toutefois artificielle car, au-delà de leurs énonciataires « légitimes ", les productions discursives sont aussi ou prioritairement destinées au chercheur. La situation de communication « habituelle » se distingue des deux précédentes dans la mesure où la performance aurait lieu même en l'absence du chercheur.

6. Pour des raisons de discrétion, je ne donnerai pas ici le nom des chanteuses. Je désignerai les solistes par une lettre marquant leur groupe d'appartenance (Grande ou Petite épouse) et un nombre de 1 à 6 qui permet de distinguer leur ordre d'apparition dans le chant.

7. Les descendants de captifs issus d'une famille restée attachée durant trois générations à une même famille étaient considérés comme des "captifs de case" (horso); ils ne pouvaient être vendus ni maltraités et se spécialisaient dans une activité, devenant «artisans » (du cuir, du fer, etc.). Les garaasa sont par conséquent ceux qui travaillent le cuir et les femmes sont particulièrement réputées pour leurs chants.

8. Par rapport à la capitale, Niamey, les cantons zarma de Hamdallaye, Tondikiwindi ainsi que Liboré et le canton songhay de Gotheye sont respectivement situés à : 30km environ au nord-est, plus de $300 \mathrm{~km}$ au nord, $15 \mathrm{~km}$ environ au sud et $80 \mathrm{~km}$ au nord-ouest.

9. Une fille de la famille «noble » de Liboré avec laquelle je suis proche et qui venait m'aider pour la prise de sons.

10. Ceux-ci sont généralement désignés par le terme générique de " chose ».

11. Dans le sens de l'ensemble du sexe féminin.

12. Cita : lézard considéré, selon les préceptes religieux, comme impur. L'eau de boisson devient ainsi inconsommable, quand il y choit (ce qui arrive puisque, comme nos souris, il fréquente les habitations). Il peut, d'aventure, laisser tomber ses œufs dans cette eau, invisibles au fond de la jarre, et les gens consommeraient alors à leur insu cette eau dégoutante. Le lézard symbolise la verge et les œufs le sperme.

13. L'adultère féminin est d'ailleurs abordé sans condamnation particulière, au contraire des attitudes masculines citées ci-dessus.

14. Essentiellement périphrases, synecdoques, métaphores et antiphrases. 
15. On retrouve ici le constat de LAUNAY (2006, p. 805) au sujet de ce qu'il nomme les « ceremonial joking » : "Among Dyula, weddings and especially funerals of elders are rituals occasions during joking is a predictable feature. Senanku, worosso, grandchildren all have scripted parts to play, though they always have a certain amount of latitude in which to improvise. But the outcome of the drama is always uncertain. It may serve to deflate the pretensions on the relatively rich and powerful, or alternatively an occasion on which to demonstrate their largesse and their savoirfaire ».

16. Je tiens à remercier Safia AMADOU qui m'a été d'une grande aide pour la transcription et la traduction de cette performance ainsi que Jean DERIVE, Alice DEGORCE, Cécile LEGUY, Marie LORILLARD et Christiane SEYDOU pour leurs précieux conseils.

17. Ce poison est un raticide sous forme de poudre, utilisé dans les villages pour tuer les poux qu'on trouve dans les cheveux.

18. Précisons que la laideur, comme les défauts moraux ou la pauvreté, fait partie des plaisanteries mineures dans les relations à plaisanterie malinké, alors que la sexualité est un thème beaucoup plus grave.

19. Le wanzam est le barbier 'traditionnel', mais aussi celui qui circoncisait les jeunes hommes autrefois.

20. Non numérotée.

21. Variante songhay.

22. (P1) Iri se ye fu iri ga goro iri ga mimiri iri ga goro kaaniyew haw kaani zu da iri ma kaani zu a sinda taali.

Nous ne retournerons pas chez nous nous resterons nous resterons nous resterons nous sentirons le plaisir nous savourerons le plaisir si nous savourons le plaisir il n'y aura pas de problème. (v. 66)

23. (P1) [...] ay ma a kolli ay ma a ganday ay ma a mooru ay ma a ganday.

Je la tire je l'enlace je la caresse je l'enlace. (v. 58)

24. Il s'agit d'un emprunt au hausa, uwar gida, qui signifie « première épouse ».

\section{RÉSUMÉS}

Au moment du remariage d'un homme, on observe - chez les Songhay-Zarma du Niger - un rituel spécifique aux mariages polygames, le marcanda, où les femmes, divisées entre "grandes » et " petites » épouses, se lancent dans une joute verbale d'insultes, puis chantent ensemble. Au sein de ce rituel, des chanteuses d'origine captives peuvent parfois venir chanter des chants grivois. Elles y évoquent ce dont on ne parle pas dans la vie quotidienne : la sexualité. Dans cet article, j'analyserai - sur la base d'une approche énonciative et pragmatique - le dernier chant d'une performance qui en totalise trente-deux. Celui-ci est particulièrement intéressant, car il débouche sur une altercation qui nous permettra de montrer comment ces chants de captives obéissent à des normes, bien qu'ils s'inscrivent dans la transgression, et comment cet espace transgressif, s'il est délimité, est sans cesse renégocié.

There is, when a man remarries among the Songhay-Zarma of Niger, a specific ritual for polygamous marriages, called the marcanda, in which women, divided into " big " and « little " wives, engage in a verbal joust and then sing together. During this ritual, singers of captive descent sometimes perform saucy songs. They evoke what is never spoken about in everyday life: 
sexuality. In this paper I will analyze, from an enunciative and pragmatic perspective, the last song from a performance of thirty-two. This song is interesting for it leads to a quarrel that shows how norms are followed in these captive songs, even though they are transgressive, and how this transgressive space, while bound, is constantly renegociated.

INDEX

Mots-clés : chant, énonciation, joute verbale, mariage, Niger, norme, polygamie, pragmatique, Songhay-Zarma, subversion, transgression

Keywords : enunciation, marriage, Niger, norm, polygamy, pragmatics, song, Songhay-Zarma, subversion, transgression, verbal joust

\section{AUTEUR}

SANDRA BORNAND

CNRS, UMR 8135 - LLACAN (Langage, Langues et Cultures d'Afrique Noire) 